\section{Prophylaxis and therapy of viral infections in pediatric patients treated for malignancy}

\author{
Maria Licciardello, ${ }^{1}$ Anna Pegoraro,2 \\ Simone Cesaro² \\ 1Pediatric Hematology Oncology, \\ University of Catania; 2 Pediatric \\ Hematology Oncology, Azienda \\ Ospedaliera Universitaria Integrata, \\ Verona, Italy
}

\begin{abstract}
Infections are still an important cause of mortality and morbidity in pediatric cancer patients. Most of the febrile episodes in immunocompromised patients are classified as a fever of unknown origin (FU0) while bacteria are the more frequent causes of documented infections. Viral infections are also feared during chemotherapy but less data are available on their incidence and morbidity. We reviewed the literature on incidence, morbidity, and mortality of viral infections in children undergoing chemotherapy and discussed the evidence concerning the prophylaxis and the therapy.
\end{abstract}

\section{Introduction}

During the last decades, survival rates in pediatric cancer have continuously increased. Infections are an important cause of morbidity and mortality for children with malignancy. Infectious complications not only contribute to mortality but also prolong hospitalisation, delay chemotherapy delivery, affect quality of life and increase healthcare utilization and costs.

The most frequent causal pathogens of febrile neutropenia (FN) are bacteria or fungi; they are identified and confirmed by culture in only $25-30 \%$ of the cases. In other $15-25 \%$ of patients with FN, bacterial or fungal infections are suspected on clinical findings. The remaining $50 \%$ of cases are classified as fever of unknown origin (FUO) and may be caused by other pathogens, namely virus, that are more difficult to detect by conventional methods. ${ }^{1}$

Generally viral infections are suspected on the basis of clinical data, laboratory findings and lack of other identified sources of fever; nevertheless, often it is difficult to confirm a suspected viral etiology on the basis of clinical findings with the isolation of the virus. ${ }^{2}$

Understanding the prevalence and the characteristics of viral infections can improve the treatment of febrile episodes in cancer patients. Unfortunately, very few studies on viral diseases in pediatric cancer patients have been published.

The respiratory viruses are one of the more frequent cause of viral infection in cancer patients and are implicated in the commonest seasonal-related community-acquired infections. Christensen observed in a prospective follow-up study all the febrile episodes during 12 months in a pediatric oncology department; nineteen viral infections were diagnosed in 250 febrile episodes. By molecular diagnosis, he increased the microbiologic verification of infections to $35 \%$. Overall, viral infections were rare but he reported a fatal complication caused by respiratory syncytial virus (RSV). Moreover he found a lower number of febrile episodes in solid tumor patients while the viral infections were more frequent in this group than in patients with hematologic malignancies. $^{3}$

Katsimpardi et al collected retrospectively all infectious episodes in children with acute lymphoblastic leukemia (ALL) from a single institution, i.e. 610 episodes over 6 years. The objective was to assess the type, frequency, severity and outcome of all infectious episodes. Viral infections were diagnosed in about $10 \%$ of episodes. The most frequent diagnosis were varicella-zoster (VZV) and herpes simplex virus (HSV) infections. Most of the severe infections occurred during the induction therapy (bacteremia and FU0), while viral infections were more common during maintenance treatment. ${ }^{4}$

In another study, viral pathogens were identified in $34 \%$ of the episodes of FN. ${ }^{5}$ This study compared patients with FUO with patients affected by viral infections; patients with FU0 had shorter duration of fever and hospitalisation; only few cases developed clinical complication. This study described less FUO (53\% of total FN) and more diagnoses of viral infections compared with other similar observational studies. ${ }^{6}$ The majority of bacteremia findings diagnosed by blood culture were detected within 24 hours and most FU0 (66\%) had fever for less than 24 hours. The authors emphasize that increasing detection of viral infections can improve treatment of FN, establishing different, risk based approaches for management of fever in pediatric cancer patients.

Despite the paucity of studies on epidemiological aspects of viral diseases in children undergoing chemotherapy, literature reports many case descriptions of severe or lethal viral infections. These reports are in opposition with the common opinion that viruses are quite harmless in cancer patients. ${ }^{7-11}$ We reviewed literature on incidence, morbidity and mortality of viral infections in children undergoing chemotherapy and discussed the evidence concerning the prophylaxis and the therapy.
Correspondence: Maria Licciardello, Pediatric Hematology Oncology, Azienda Ospedaliera Universitaria Policlinico Vittorio Emanuele, via Santa Sofia 78, 95123, Catania, Italy.

Tel: +39.095-3782469 - Fax: +39.095-3781453.

E-mail: marialicciar@yahoo.it

Key words: chemotherapy, pediatric malignancy, viral infections.

Received for publication: 29 November 2010. Accepted for publication: 22 December 2010.

This work is licensed under a Creative Commons Attribution 3.0 License (by-nc 3.0).

(C) Copyright M. Licciardello et al., 2011

Licensee PAGEPress, Italy

Pediatric Reports 2011; 3:e5

doi:10.4081/pr.2011.e5

\section{Methods}

For the literature search we used the key words prophylaxis, viral infections, pediatric malignancy and therapy, viral infections, pediatric malignancy. The literature search was limited to English language papers and the period analyzed was from 1 January 1990 to 31 July 2010. Reference list of papers selected by literature search was also used as source, if necessary. The results of the search were first discussed and scored by the authors and then presented for the final discussion and approval to a expert meeting held in Florence on 12 and 13 October 2010 where the representatives of centers belonging to Italian Association of Pediatric Hematology Oncology (AIEOP) attended. The scoring system proposed by Infectious Diseases Society of America was adopted to define the quality of evidence and strength of recommendations (Table 1).

\section{Varicella-Zoster virus}

Like other herpesvirus infection, varicellazoster virus (VZV) can remain latent after primary infection; immunosuppression caused by chemotherapy increases the likelihood of reactivation. Before the introduction of acyclovir and hyper-immune immunoglobulin, mortality caused by disseminated varicella was high. Moreover, in ALL patients who had developed previously VZV, Herpes zoster may occur up to 25\%. Prophylaxis with VZV immunisation has decreased this complication.

Leukemic patients and parents should be informed about VZV transmission and advised of strategies on how to avoid exposure (AIII). Family members, household contacts and healthcare workers known to be VZV-seronegative or children with no history of VZV infection should be given varicella vaccine (BIII). 
All patients with varicella or disseminated zoster should be placed under airborne and contact isolation. The isolation should continue as long as the rash remains vesicular and until all lesions are crusted (BIII).

Passive immunization with human Varicella-zoster immunoglobulin (VZIG) should be given as soon as possible after exposure $(<96 \mathrm{~h})$ to VZV-seronegative leukemic patients on chemotherapy (AII). Where passive immunization is not available, post-exposure prophylaxis with acyclovir starting 7-9 days after exposure is recommended (20 $\mathrm{mg} / \mathrm{kg}$ four times daily for 7 days - max 800 $\mathrm{mg} /$ dose) (AIII). If a second exposure occurs more than 21 days after a dose of passive immunization or after the administration of the antiviral prophylaxis, a prophylaxis should be readministered (CIII).

In case of disease, antiviral treatment reduces the duration of viral replication and clinical manifestation. Patients treated for leukemia who have a varicella-like rash should be started immediately on i.v. acyclovir 20 $\mathrm{mg} / \mathrm{kg}$ every $8 \mathrm{~h}$ for $5-10$ days (AI). Therapy (i.v./oral) should be given for at least 7 days and to be continued until 2 days after all lesions are crusted (AI). Vigilance for the possibility of visceral VZV disease without mucocutaneous manifestations (for example, in cases of encephalitis, pneumonitis or hepatitis) is needed, and i.v. acyclovir should be considered in such cases (AIII). Foscarnet (60 $\mathrm{mg} / \mathrm{kg}$ every $12 \mathrm{~h}$ i.v.) or cidofovir $(5 \mathrm{mg} / \mathrm{kg}$ once a week for 2 weeks, then (if still needed) once every 2 weeks combined with probenecide and i.v. hydration) are alternatives for anti-VZV treatment in a case of acyclovirresistant VZV infection (AIII). ${ }^{12-14}$

\section{Herpes simplex virus}

HSV (herpes simplex virus) (type $1>$ type 2) commonly cause mucocutaneous lesions in patiens with hematologic malignancies. HSV reactivation is frequent both in patients with acute leukemia during induction chemotherapy and in SCT recipients. The incidence of HSV lesions among seropositive patients during chemotherapy is high in many observational studies in adults. In a prospective cohort study in pediatric oncology patients, HSV was detected from mouth swab and blood specimen during FN; prevalence of HSV infection was low, oral HSV was associated with longer median duration of mucositis but was not associated with prolonged fever or neutropenia. It is unknown whether early intervention with acyclovir can reduce the duration of mucositis. ${ }^{15}$

Primary HSV infection in patients treated for leukemia is unusual, and antiviral drug prophylaxis is thus not recommended in HSVseronegative leukemic patients during chemotherapy (DIII).

ALL patients during the phase of induction that are HSV-seropositive may be considered for antiviral drug prophylaxis with acyclovir (level of recommendation CIII). The therapy of choice for severe mucocutaneous or visceral HSV disease is intravenous acyclovir $250 \mathrm{mg} / \mathrm{m}^{2}$ or $5 \mathrm{mg} / \mathrm{kg}$ every $8 \mathrm{~h}$ for $7-10$ days (AI). Oral acyclovir for 10 days (AI) is considered as alternative for less serious manifestations of HSV disease; for adult patients only valaciclovir p.o. for 10 days (BIII) or famciclovir p.o. for 10 days (BIII) may be considered as well. For HSV pneumonia or HSV meningitis and encephalitis, a higher dose of i.v. acyclovir $500 \mathrm{mg} / \mathrm{m}^{2}$ or $10 \mathrm{mg} / \mathrm{kg}$ every $8 \mathrm{~h}$ for $14-21$ days should be considered (CIII). ${ }^{13}$

\section{Cytomegalovirus}

Cytomegalovirus (CMV) is a significant cause of morbidity and mortality in patients who had undergone hematopoietic stem cell transplantation (HSCT) but it has increasingly reported also in leukemic patients treated with fludarabine or monoclonal antibodies (alemtuzumab). We adopted the ECIL recommendations (European Conference on Infections in Leukemia) for CMV prophylaxis in patients non-SCT (Table 2).

Table 1. Quality of evidence and Strength of recommendations according to the CDC grading system.23

\begin{tabular}{ll} 
Quality of evidence & Strength of recommendation \\
I & A \\
Evidence from at least one well-executed & Strong evidence for efficacy and \\
randomized trial & substantial clinical benefit \\
II & Strongly recommended \\
Evidence from at least one well-designed clinical & B \\
trial without randomization; cohort or case-controlled & Strong or moderate evidence for efficacy, \\
analytic studies (preferably from more than one center; & but only limited clinical benefit \\
multiple time-series studies or dramatic results from & Generally recommended \\
uncontrolled experiments) & C \\
III & Insufficient evidence for efficacy, \\
Evidence from opinion of respected authorities based & or efficacy does not outweigh possible \\
on clinical experience, descriptive studies, or reports & adverse consequences (e.g. drug toxicity \\
or interactions) from expert committees & or cost of chemoprophylaxis or alternative \\
& approaches \\
& Optional \\
& D \\
& Moderate evidence against efficacy or for \\
& adverse outcome \\
& Generally not recommended \\
& E \\
& Strong evidence against efficacy or for \\
& adverse outcome \\
& Never recommended \\
\hline
\end{tabular}

Table 2. Cytomegalovirus prophylaxis.

Immune globulin for prevention of CMV infection or disease is not recommended EII

Leukemia patients with a lesser degree of immunosuppression do not need a specified prophylactic strategy but CMV disease must be in the differential diagnosis if symptoms compatible with CMV develop

Routine monitoring and pre-emptive therapy are not considered necessary DIII

in other hematology patients

Routine antiviral prophylaxis is not recommended DIII 
Oseltamivir prophylaxis $75 \mathrm{mg}$ once daily for 8 weeks during an influenza season has been used in the prevention of influenza infection in immunocompromised children with few side-effects. It is a prospective, non-blinded, non-controlled observational study (CIII). ${ }^{19}$

There are no large clinical trials about influenza treatment. Oseltamivir can prevent progression to pneumonia and can be used in children starting from 1 year of age, Zanamivir in children aged $>7$ years of age. It is still unknown the risk to develop resistance to antiviral agents. ${ }^{20}$

\section{Respiratory syncytial virus}

Respiratory syncytial virus (RSV) is a common cause of infection in immunocompetent and immunocompromised host. The majority of patients affected by RSV and by other respiratory virus experience mild symptoms, but occasionally they can cause pneumonia and marrow suppression with consequent delay of chemotherapy administration. ${ }^{3}$

In a review on a population of children with acute myeloid leukemia (AML) prevalence of RSV infection was low, but related mortality among children with RSV infection was high. Palivizumab is a humanized monoclonal immunoglobulin used for prophylaxis in children younger than 2 years of age with chronic lung disease who require medical therapy and those born at 32 weeks gestation or earlier; its use has been suggested in some congenital heart disease. The authors don't believe that prophylactic Palivizumab is routinely indicated in children with AML because of the low risk to catch RSV infection; moreover the rarity of this infection preclude the possibility of a randomised trial (level of evidence DIII). ${ }^{9}$

About treatment, a Cochrane review on children hospitalised for RSV infection underline that it is not demonstrated a significant benefit of IVIG treatment added to supportive care compared with supportive care alone. ${ }^{21}$ The role of Ribavirin, an antiviral agent licensed for severe RSV infection, is unclear too (level of evidence, CIII).

\section{Parvovirus}

Cytopenia caused by chemotherapy is indistinguishable from that associated with infection or other causes. A prospective study detected Parvovirus B19 DNA in pediatric patients with unexpected cytopenia during chemotherapy for ALL; classical symptoms like erythema infectiosum were absent. In some situations, for example during manteinance, cytopenia can cause suspension or reduce intensity of treatment. The authors suggest screening for parvovirus B19 in pediatric patients with ALL and unexplained cytopenia. Treatment with IVIG has proven to be successful although some studies could not document a clear benefit (level of evidence CIII).22

Table 3. Cytomegalovirus therapy.

Treatment of CMV pneumonia

Antiviral therapy with ganciclovir is recommended

AII

Foscarnet can be used in place of ganciclovir

Cidofovir or the combination of foscarnet and ganciclovir can be used as second-line therapy

BII Other types of CMV disease

Either i.v. ganciclovir or foscarnet administration without the addition of immune globulin

BII is recommended

Cidofovir or the combination of i.v. ganciclovir and foscarnet can be used as second-line therapy for CMV disease

\section{Enterovirus}

In immunocompromised children, enterovirus can cause life-theatening infections such as myocarditis, meningitis and encephalitis. Early diagnosis and treatment with IVIG together with supportive care may improve the outcome. In a retrospective study, children who received high dose of IVIG ( $2 \mathrm{gr} / \mathrm{kg}$ ) developed early negative viral load versus children treated with low dose of IVIG $(400 \mathrm{mg} / \mathrm{kg}$ ) (level of evidence CIII). ${ }^{10}$

\section{References}

1. Michálek J, Horvath R. High incidence of Epstein-Barr virus, cytomegalovirus and human herpesvirus 6 infections in children with cancer. BMC Pediatr 2002;2:1.

2. Yee-Guardino S, Gowans K, Yen-Lieberman $\mathrm{B}$, et al. Beta-herpesviruses in febrile children with cancer. Emerg Infect Dis 2008;14:579-85.

3. Christensen MS, Nielsen LP, Hasle H. Few but severe viral infections in children with cancer: a prospective RT-PCR and PCRbased 12-month study. Pediatr Blood Cancer 2005;45:945-51.

4. Katsimpardi K, Papadakis V, Pangalis A, et al. Infections in a pediatric patient cohort with acute lymphoblastic leukemia during the entire course of treatment. Support Care Cancer 2006;14:277-84.

5. Hakim H, Flynn PM, Knapp KM, et al. Etiology and clinical course of febrile neutropenia in children with cancer. J Pediatr Hematol Oncol 2009;31:623-9.

6. Castagnola E, Fontana V, Caviglia I, et al. A prospective study on the epidemiology of febrile episodes during chemotherapyinduced neutropenia in children with cancer or after hemopoietic stem cell transplantation. Clin Infect Dis 2007;45:1296304.

7. Caselli D, Carraro F, Castagnola E, et al. Morbidity of pandemic H1N1 influenza in children with cancer. Pediatr Blood Cancer 2010;55:226-8.
8. Wittekindt B, Berger A, Porto L, et al. Human herpes virus-6 DNA in cerebrospinal fluid of children undergoing therapy for acute leukaemia. Br J Haematol 2009;145:542-5.

9. Sung L, Alonzo TA, Gerbing RB, et al. Children's Oncology Group. Respiratory syncytial virus infections in children with acute myeloid leukemia: a report from the Children's Oncology Group. Pediatr Blood Cancer 2008;51:784-6.

10. Moschovi MA, Katsibardi K, Theodoridou $\mathrm{M}$, et al. Enteroviral infections in children with malignant disease: a 5 -year study in a single institution. J Infect 2007;54:387-92.

11. Spacca B, Mallucci C, Riordan A, et al. HSV encephalitis in a child with brain stem glioma: a rare complication of therapy. Case report and review of the neurosurgical literature. Childs Nerv Syst 2007;23: 1347-50.

12. Kempf W, Meylan P, Gerber S, et al. Swiss recommendations for the management of varicella zoster virus infections. Swiss Med Wkly 2007;137:239-51.

13. Styczynski J, Reusser P, Einsele $\mathrm{H}$, et al. Second European Conference on Infections in Leukemia. Management of HSV, VZV and EBV infections in patients with hematological malignancies and after SCT: guidelines from the Second European Conference on Infections in Leukemia. Bone Marrow Transplant 2009;43:757-70.

14. Bailey LC, Reilly AF, Rheingold SR. Infections in pediatric patients with hematologic malignancies. Semin Hematol 2009;46:313-24.

15. Ramphal R, Grant RM, Dzolganovski B, et al. Herpes simplex virus in febrile neutropenic children undergoing chemotherapy for cancer: a prospective cohort study. Pediatr Infect Dis J 2007;26:700-4.

16. Ljungman P, de la Camara R, Cordonnier C, et al. European Conference on Infections in Leukemia. Management of CMV, HHV-6, HHV-7 and Kaposi-sarcoma herpesvirus (HHV-8) infections in patients with hematological malignancies and after SCT. Bone Marrow Transplant 2008;42:227-40.

17. Nigro G, Krzysztofiak A, Bartmann U, et al. 
Ganciclovir therapy for cytomegalovirusassociated liver disease in immunocompetent or immunocompromised children. Arch Virol 1997;142:573-80.

18. Jancel T, Penzak SR. Antiviral therapy in patients with hematologic malignancies, transplantation, and aplastic anemia. Semin Hematol 2009;46:230-47.

19. Chik KW, Li CK, Chan PK, et al. Oseltamivir prophylaxis during the influenza season in a paediatric cancer centre: prospective observational study. Hong Kong Med J 2004;10:103-6.
20. Whitley RJ, Monto AS. Prevention and treatment of influenza in high-risk groups: children, pregnant women, immunocompromised hosts, and nursing home residents. J Infect Dis 2006;194:S133-8.

21. Fuller H, Del Mar C. Immunoglobulin treatment for respiratory syncytial virus infection. Cochrane Database Syst Rev 2006; CD004883.

22. Lindblom A, Heyman M, Gustafsson I, et al. Parvovirus B19 Infection in children with Acute Lymphoblastic Leukemia is associated with cytopenia resulting in prolonged interruptions of chemotherapy. Clin Infect Dis 2008;46:528-36.

23. Centers for Disease Control and Prevention. Guidelines for preventing opportunistic infections among hematopoietic stem cell transplant recipients. Recommendations of CDC, the Infectious Disease Society of America, and the American Society of Blood and Marrow Transplantation. MMWR Recomm Rep 2000;49:1-125. 